\title{
Minireview \\ Selective bladder preservation for muscle-invasive transitional cell carcinoma of the urinary bladder
}

\author{
MD Michaelson*,', WU Shipley', NM Heney', AL Zietman' and DS Kaufman' \\ 'Departments of Hematology/Oncology, Radiation Oncology and Urology, Massachusetts General Hospital Cancer Center, Harvard Medical School, \\ Boston, MA, USA
}

Invasive transitional cell carcinoma (TCC) of the urinary bladder is traditionally treated with radical cystectomy. This approach results in great morbidity and lifestyle changes, and approximately half of the patients treated in this way will experience recurrent TCC despite surgery. An alternative approach using selective bladder-preservation techniques incorporates transurethral resection of bladder tumours, radiation therapy, and chemotherapy. Over the past 20 years, international experience has demonstrated that this approach is feasible, safe, and well tolerated. Furthermore, the long-term outcomes of overall survival and disease-free survival compare favourably with the outcomes from radical cystectomy. The most important predictor of response is stage, with significantly higher long-term survival in patients with T2 disease. Another important positive predictor of complete response to therapy is the ability of the urologic oncologist to remove all visible tumour through a transurethral approach prior to initiation of radiation therapy. A negative predictive factor is the presence of hydronephrosis, and age and gender do not affect disease-free survival. The majority of patients who enjoy long-term survival do so with an intact native bladder. Quality of life studies have demonstrated that the retained bladder functions well in nearly all of these patients. Selective bladder preservation will not entirely take the place of radical cystectomy, but should be offered as an important alternative to patients newly diagnosed with muscle-invasive TCC. British Journal of Cancer (2004) 90, 578-58I. doi:I0.I038/sj.bjc.660I580 www.bjcancer.com (c) 2004 Cancer Research UK

Keywords: bladder cancer; radiation therapy; chemotherapy; radical cystectomy; cisplatin

Radical cystectomy has been the standard treatment for muscle invasive transitional cell carcinoma (TCC) of the bladder. Radical surgery results, historically, in a long-term survival rate of only 40-60\% (Pagano et al, 1991; Waehre et al, 1993). More recent analysis of radical cystectomy at a single institution demonstrated a disease-specific survival of $67 \%$ with a median follow-up of 65 months, and long-term overall survival of $45 \%$ (Dalbagni et al, 2001). Another single-institution series stratified patients by pathologic stage, and found a recurrence-free survival of $89 \%$ in pathologic $\mathrm{T} 2$, node-negative tumours, $50 \%$ in T4 node-negative tumours, and $35 \%$ in patients with node-positive tumours (Stein et al, 2001). The negative impact that urinary diversion has on lifestyle is part of the impetus for a search for alternatives.

Cystoscopic tumour resection alone or radiation therapy alone provide inferior outcomes to radical cystectomy, with only $20-$ $40 \%$ local control rates in muscle invasive TCC. However, an accumulation of international experience over the past two decades has established that trimodality bladder preservation treatment is a legitimate alternative in selected cases of patients with muscle-invasive TCC. The algorithm for this approach is initial cystoscopic resection of as much bladder tumour as is safely possible, followed by a combination of bladder irradiation with

*Correspondence: Dr MD Michaelson;

E-mail: dmichaelson I@partners.org

Received 5 September 2003; revised 13 November 2003; accepted 20 November 2003 concurrent radiosensitising chemotherapy, followed by adjuvant chemotherapy. Active cystoscopic surveillance of the bladder is maintained throughout the treatment period, and radical cystectomy is advised in the event of persistent invasive disease. Although randomised studies comparing this approach to surgery have not been performed, survival outcomes with selective bladder preservation are comparable to outcomes from radical cystectomy in comparable patients. This review will focus on studies of selective bladder-preserving treatment of muscle-invasive TCC, as well as on quality of life measures in patients who have undergone this treatment.

\section{INTERNATIONAL EXPERIENCE WITH SELECTIVE BLADDER PRESERVATION}

Selective bladder preservation for muscle-invasive TCC in North America has occurred primarily under the auspices of the Radiation Therapy Oncology Group (RTOG) and the National Bladder Cancer Group. A series of six consecutive RTOG clinical trials has been completed to date. The initial study RTOG 85-12 treated 42 patients with daily radiation therapy and concurrent cisplatin, and reported greater than 50\% 5-year survival (Tester et al, 1993). The approach was feasible, well tolerated by patients, and resulted in $42 \%$ long-term survival with an intact bladder. Subsequent studies conducted by the RTOG have explored numerous additional questions, including the addition of other chemotherapy agents to cisplatin, including 5-fluorouracil (5FU) and paclitaxel, the feasibility of outpatient treatment with this 
approach, the utility of neoadjuvant or adjuvant chemotherapy, the use of hyperfractionated radiation therapy, and others. These studies will be further described below.

Concurrently, a number of European groups engaged in pioneering efforts in bladder-preserving approaches to treatment. The University of Paris treated 54 patients with transurethral surgery, followed by concomitant radiation and chemotherapy (Housset et al, 1993). In this study, 5FU was added to cisplatin, and twice daily radiation was employed. Treatment was well tolerated, and disease-free survival was $62 \%$ at 3 years.

The University of Erlangen reported on their 10-year experience of 79 patients treated with transurethral bladder resection followed by daily radiation with concurrent cisplatin (Dunst et al, 1994). Results were similar to those of the RTOG and the University of Paris, with 52\% 5-year overall survival and $41 \%$ survival with intact bladder. Their experience was expanded in a subsequent report in which 162 patients were treated with either cisplatin or carboplatin (Sauer et al, 1998). The overall 5-year survival was $55 \%$, and $44 \%$ of total patients survived long term with an intact bladder. Patients treated with carboplatin did not have as favourable an outcome as those treated with cisplatin, although the numbers in each group were too small for meaningful statistical comparison. A subsequent study demonstrated that the combination of cisplatin and 5FU for radiosensitising chemotherapy was safe, tolerable, and efficacious (Rodel et al, 2002b).

A common theme among these studies was the use of salvage radical cystectomy when necessary. Surveillance cystoscopy was performed following combination treatment in the European studies, whereas the RTOG and Massachusetts General Hospital (MGH) studies employed an interval cystoscopy after an induction' period of treatment to assess the response. Patients with less than complete response to combination therapy were referred for radical cystectomy. The complete response rate found at cystoscopy following induction or completed therapy in these studies was generally in the range of $65-75 \%$. Patients with only carcinoma in situ, or with superficial cancer at a new site, were included in the group of complete responders, whereas patients with any muscle-invasive bladder cancer, or with superficial cancer at the original site, were referred for surgery.

In the largest series yet reported, the University of Erlangen recently compiled their data on 415 patients treated with selective bladder preservation over an 18-year period, including the 162 patients described in their prior study (Rodel et al, 2002a). Of the 415 patients, 89 had stage T1 disease only, and the remainder had T2-T4. The large majority had no evidence of lymph node metastases. Over 100 patients were treated with radiation alone, whereas 289 were treated with concurrent chemotherapy and radiation. Radiosensitising chemotherapy consisted of cisplatin (145), carboplatin (95), or cisplatin and 5FU (49). Results were reported for all 415 patients as a group, which somewhat obscured the results for patients treated with combination therapy. The complete response rate at interval cystoscopy was significantly higher among patients treated with combination therapy than among patients treated with radiation alone $(P=0.001)$. The overall 5-year survival including T1 patients was 51\%, and survival with an intact bladder was $42 \%$. The authors also found that initial tumour response was a strong prognostic factor for diseasespecific survival and overall survival.

It is important to emphasise the role of transurethral debulking (TURB) in combined modality bladder-preservation treatment. The complete response rate of $65-75 \%$ at interval cystoscopy reflects not only the effect of concurrent radiation and chemotherapy, but also the effect of TURB. It is likely that TURB alone would result in a complete response in some patients, and, even in those with persistent microscopic disease after resection, the debulking effect of TURB probably enhances the efficacy of concurrent radiation and chemotherapy. Ability to completely resect visible tumour was a strong predictor of complete response as well as overall survival in univariate and multivariate analyses (Shipley et al, 1987; Rodel et al, 2002a).

Another critical surgical point is the essential role of salvage cystectomy in incomplete responders to combined modality therapy. Of 190 patients treated with selective bladder preservation, 66 patients $(35 \%)$ ultimately underwent radical cystectomy for incomplete response to therapy or recurrent invasive tumour (Shipley et al, 2002). The 5-year disease-specific survival rate for these patients was $57 \%$ for stage T2 and $42 \%$ for stage $\mathrm{T} 3-\mathrm{T} 4 \mathrm{a}$, confirming that salvage surgery is potentially curative therapy for many patients in this program. Importantly, no patients have required cystectomy to treat radiation-induced bladder toxicity.

\section{RTOG STUDIES}

Since the initial publication of RTOG 85-12, this group has completed a series of clinical trials examining combined modality therapy (Table 1). A major effort was undertaken to determine the role of neoadjuvant chemotherapy in studies 88-02 and, in a randomised phase III trial, in 89-03 (Tester et al, 1996; Shipley et al, 1998). The majority of patients who relapse following definitive local therapy have distant, rather than local, relapse, indicating the likelihood of microscopic metastatic disease at the time of local therapy. Thus, the use of systemic chemotherapy is employed in an attempt to eradicate such microscopic disease. However, in 89-03, treating patients with two cycles of MCV chemotherapy (methotrexate, cisplatin, vinblastine) prior to combination therapy failed to improve survival or local tumour eradication. At a median follow-up of 5 years, the overall survival was $48 \%$ in patients treated with MCV, and $49 \%$ in the untreated arm. Cystoscopic complete response was $61 \%$ in the $\mathrm{MCV}$ arm, and $55 \%$ in the control arm. At 5 years, distant metastases were present in $35 \%$ of the patients treated with neoadjuvant MCV, and $43 \%$ of the untreated patients. None of these differences approached statistical significance. Moreover, because of toxicity, the protocolcompletion rate for the MCV arm was only $67 \%$. As a result, some subsequent studies employed adjuvant rather than neoadjuvant chemotherapy. Numerous randomised trials have explored

Table I RTOG bladder-preservation studies

\begin{tabular}{|c|c|c|c|c|c|c|}
\hline RTOG number & $\begin{array}{l}\text { Adjuvant/neoadjuvant } \\
\text { therapy }\end{array}$ & Radiation therapy & $\begin{array}{l}\text { Radiosensitising } \\
\text { chemotherapy }\end{array}$ & \# of patients & $\begin{array}{l}\text { 5-year } \\
\text { survival }\end{array}$ & Reference \\
\hline $85-12$ & None & Daily & Cisplatin & 42 & $52 \%$ & (Tester et al, 1993) \\
\hline $88-02$ & Neoadjuvant MCV & Daily & Cisplatin & 91 & $62 \%$ & (Tester et al, 1996) \\
\hline $89-03$ & +Neoadjuvant MCV & Daily & Cisplatin & 123 & $49 \%$ & (Shipley et al, 1998) \\
\hline $95-06$ & None & Hypofractionated & Cisplatin+5FU & 34 & $N / A$ & (Kaufman et al, 2000) \\
\hline $97-06$ & Adjuvant MCV & Twice daily & Cisplatin & 52 & N/A & (Hagan et al, 2003) \\
\hline $99-06$ & Adjuvant GC & Twice daily & Cisplatin+paclitaxel & 84 & N/A & - \\
\hline
\end{tabular}

MCV - methotrexate, cisplatin, vinblastine; 5FU - 5-fluorouracil; GC - gemcitabine, cisplatin; N/A - not available. 
the use of neoadjuvant or adjuvant chemotherapy together with radical cystectomy, a topic beyond the scope of this review (Bajorin, 2001; Raghavan et al, 2002; Sternberg, 2002; Grossman et al, 2003).

In subsequent RTOG studies, a number of modifications were made to the radiation treatment. High-dose hypofractionation was employed in 95-06, together with both cisplatin and 5FU for radiosensitisation (Kaufman et al, 2000). Seven of 34 patients (21\%) experienced grade 3 or 4 haematological toxicity, though no deaths resulted. No patients required cystectomy for radiation toxicity, despite the high-dose hypofractionation. Complete response was seen in $67 \%$ of patients, and 3-year overall survival was $66 \%$. The RTOG then turned to conventional twice-daily hyperfractionation in 97-06 and subsequent studies. In 97-06, a total of $45.6 \mathrm{~Gy}$ was delivered to the pelvis and bladder, and $64.8 \mathrm{~Gy}$ to the bladder tumour. Individual fractions were $1.8 \mathrm{~Gy}$ to the pelvis with a boost of $1.6 \mathrm{~Gy}$ to the bladder tumour during induction treatment, twice a day for 12 days, and then $1.5 \mathrm{~Gy}$ fractions to both during consolidation treatment, twice a day for 8 days (Hagan et al, 2003). In 99-06, radiation was delivered in a similar fashion with a boost to the whole bladder, and an additional boost to the bladder tumour. The full results of these studies are not yet published.

Initial RTOG studies required hospitalisation of patients to deliver the combined modality treatment. However, since 95-06, all treatments have been administered in the outpatient clinics, with only rare hospitalisations for complications of therapy. This approach has been feasible and safe, and has greatly increased the appeal of combined modality therapy.

\section{PREDICTORS OF OUTCOME}

Initial clinical stage continues to be the most important predictor of overall survival in patients treated for muscle-invasive bladder cancer. In a series of 190 patients treated with selective bladder preservation at the $\mathrm{MGH}$, 5-year overall survival and diseasespecific survival were 62 and $63 \%$, respectively, for stage T2, and 47 and $53 \%$ for stage T3-T4a (Shipley et al, 2002). Similarly, stage was an important predictor of survival with an intact bladder. The 5 -year disease-specific survival with an intact bladder was $57 \%$ for patients with stage T2, but only $35 \%$ for patients with stage T3T4a. In the series from the University of Erlangen, 5-year overall survival was $75 \%$ for stage T1, $56 \%$ for T2, $44 \%$ for $\mathrm{T} 3$, and $17 \%$ for T4 (Rodel et al, 2002b).

The presence or absence of hydronephrosis at the time of diagnosis has also affected treatment success. Of the 190 patients reviewed at the MGH, 27 had initial hydronephrosis (Shipley et al, 2002). Their likelihood of complete response at the first interval cystoscopy was $37 \%$, compared with $68 \%$ in patients without hydronephrosis $(P=0.002)$. Ultimately, $60 \%$ of these patients underwent radical cystectomy, compared with $31 \%$ of the other patients $(P=0.004)$. Despite the low total numbers of patients with hydronephrosis, this factor approached statistical significance for predicting survival in multivariate analysis. The 5 -year overall and disease-specific survivals were 48 and $53 \%$ for patients with hydronephrosis, compared with 55 and $64 \%$ for patients without hydronephrosis. As a result of this finding, patients with initial hydronephrosis are generally excluded from bladder-preservation protocols.

The initial response to treatment does not predict survival outcome, but is an independent predictor of successful bladder preservation. Not surprisingly, advanced age predicts poorer overall survival for patients treated with selective bladder preservation (Rodel et al, 2002b; Shipley et al, 2002). However, there is no clear effect on disease-specific survival, suggesting that age may not impact on the success of this treatment approach. Gender has not been found to affect outcome.

\section{COMPARISON WITH TREATMENT OUTCOMES FROM CONTEMPORARY CYSTECTOMY SERIES}

The discrepancy between clinical staging and pathologic staging is an important confounding factor in comparing results of radical cystectomy with results of bladder preservation. Since clinical staging is more likely to understage the extent of disease, there is an outcome bias in favour of surgical series that benefit from pathologic staging. Nevertheless, outcomes from bladder preservation treatment compare favourably to outcomes reported from contemporary surgical series.

Two large, single-institution surgical series have been recently published. The University of Southern California reported on 1054 patients undergoing radical cystectomy (Stein et al, 2001). Of these, 633 patients had pathologic stage T2-T4a, and their 5-year overall survival rate was $48 \%$. A second series, from Memorial Sloan Kettering Cancer Center, reported a 5-year overall survival rate of $36 \%$ in patients with T2-T4 tumours (Dalbagni et al, 2001). Finally, a recent Intergroup study examining neoadjuvant chemotherapy in patients undergoing radical cystecomy for muscleinvasive bladder cancer reported a 5-year overall survival of $50 \%$ (Grossman et al, 2003). In patients who underwent cystectomy without receiving chemotherapy, the 5-year survival was only $43 \%$. Thus, even contemporary surgical series demonstrate a high rate of disease recurrence for muscle-invasive disease, with cure rates no higher than those that have been observed in current selective bladder-preserving approaches (see Table 1). Comparison is hindered by the lack of a phase III, randomised trial of the two approaches. Unfortunately, cooperation for such an endeavour has not yet been forthcoming from the different involved disciplines.

\section{QUALITY OF LIFE OUTCOMES}

There are legitimate concerns among physicians regarding the long-term effects of high-dose bladder radiation on bladder function, as well as the potential harmful effects to the bowel and to erectile function in men. In earlier cross-sectional studies from Italy and Sweden, over $74 \%$ of patients reported good longterm urinary function, and low levels of patient-reported systems (Caffo et al, 1996; Henningsohn et al, 2002). A recent study from the MGH has studied the quality of life and urodynamics in a comprehensive fashion among 49 patients who had completed bladder preservation therapy a median of 6.3 years earlier (Zietman et al, 2003). In all, 32 patients agreed to undergo formal urodynamic evaluation. Of these, 24 were judged to have bladders with normal function. The median volume voided was $284 \mathrm{~cm}^{3}$ (range $125-630 \mathrm{~cm}^{3}$ ), and median bladder capacity was $400 \mathrm{~cm}^{3}$ (range 221-747). Five of the 18 men studied had evidence of reduced flow. Three had post-void residual of greater than $100 \mathrm{~cm}^{3}$, and two of these had urodynamic evidence of bladder outlet obstruction. Thus, impaired urinary function may have been due to external factors and not to impaired bladder function per se.

One-fifth of all patients in the study reported some degree of incontinence within the preceding week, and women were twice as likely as men to report incontinence. Mild to moderate bowel symptoms were reported by $20 \%$ of men and $27 \%$ of women. The symptom most frequently reported was rectal urgency. A total of $14 \%$ reported moderate or greater symptoms, but no patients reported severe or very frequent bowel symptoms. In two prior studies, 10 and $32 \%$ of patients complained of moderate or greater bowel symptoms. Sexual function among men was remarkably intact, with $8 \%$ reporting dissatisfaction with their sex life, $33 \%$ reporting neither satisfaction nor dissatisfaction, and 59\% reporting satisfaction (Zietman et al, 2003). Sildenafil was used by $16 \%$ of men, perhaps accounting in part for the favourable numbers compared to earlier studies, in which only $25-40 \%$ of men retained adequate sexual function (Caffo et al, 1996; Henningsohn et $a l, 2002)$. The numbers in this study compare favourably to 
patients who undergo radiation for prostate cancer. No data were available for sexual function among women in this study.

The major conclusions of this recent study from the MGH were that normal bladder function was preserved in the majority of patients with an intact bladder years after bladder preservation therapy, that about one-fifth of patients complained of mild to moderate bowel symptoms, and that one-fifth of patients suffered from occasional urinary incontinence.

\section{CONCLUSIONS}

The use of bladder-preservation therapy for muscle-invasive TCC of the bladder is a valid alternative to radical cystectomy in selected cases. Long-term efficacy is comparable to radical cystectomy, with the advantage of preserving excellent bladder function in the majority of long-term survivors. Contemporary protocols utilise a combination of transurethral tumour resection, concurrent radiation and radiosensitising chemotherapy, and often adjuvant chemotherapy. These approaches require close coordination among surgical, radiation, and medical oncologists. Treatments are well tolerated, and are carried out in the outpatient setting. Future investigation will optimise the use of systemic therapy during selective bladder preservation, and will explore the role for rationally targeted biologic agents in the management of locally advanced bladder cancer.

\section{REFERENCES}

Bajorin DF (2001) Plenary debate of randomized phase III trial of neoadjuvant MVAC plus cystectomy versus cystectomy alone in patients with locally advanced bladder cancer. J Clin Oncol 19: 17S-20S

Caffo O, Fellin G, Graffer U, Luciani L (1996) Assessment of quality of life after cystectomy or conservative therapy for patients with infiltrating bladder carcinoma. A survey by a self-administered questionnaire. Cancer 78: 1089-1097

Dalbagni G, Genega E, Hashibe M, Zhang ZF, Russo P, Herr H, Reuter V (2001) Cystectomy for bladder cancer: a contemporary series. J Urol 165: $1111-1116$

Dunst J, Sauer R, Schrott KM, Kuhn R, Wittekind C, Altendorf-Hofmann A (1994) Organ-sparing treatment of advanced bladder cancer: a 10-year experience. Int J Radiat Oncol Biol Phys 30: 261-266

Grossman HB, Natale RB, Tangen CM, Speights VO, Vogelzang NJ, Trump DL, deVere White RW, Sarosdy MF, Wood Jr DP, Raghavan D, Crawford ED (2003) Neoadjuvant chemotherapy plus cystectomy compared with cystectomy alone for locally advanced bladder cancer. $N$ Engl J Med 349: $859-866$

Hagan M, Winter K, Kaufman D, Wajsman Z, Zietman A, Heney N, Toonkel L, Jones C, Roberts J, Shipley W (2003) RTOG 9706: Initial report of a phase I/II trial of selective bladder-conservation employing TURB, twice-daily accelerated irradiation sensitized with cisplatin followed by adjuvant MCV combination chemotherapy. Int J Radiat Oncol Biol Phys, in press

Henningsohn L, Wijkstrom H, Dickman PW, Bergmark K, Steineck G (2002) Distressful symptoms after radical radiotherapy for urinary bladder cancer. Radiother Oncol 62: 215-225

Housset M, Maulard C, Chretien Y, Dufour B, Delanian S, Huart J, Colardelle F, Brunel P, Baillet F (1993) Combined radiation and chemotherapy for invasive transitional-cell carcinoma of the bladder: a prospective study. J Clin Oncol 11: 2150-2157

Kaufman DS, Winter KA, Shipley WU, Heney NM, Chetner MP, Souhami L, Zlotecki RA, Sause WT, True LD (2000) The initial results in muscleinvading bladder cancer of RTOG 95-06: phase I/II trial of transurethral surgery plus radiation therapy with concurrent cisplatin and 5fluorouracil followed by selective bladder preservation or cystectomy depending on the initial response. Oncologist 5: 471-476

Pagano F, Bassi P, Galetti TP, Meneghini A, Milani C, Artibani W, Garbeglio A (1991) Results of contemporary radical cystectomy for invasive bladder cancer: a clinicopathological study with an emphasis on the inadequacy of the tumor, nodes and metastases classification. J Urol 145: $45-50$

Raghavan D, Quinn D, Skinner DG, Stein JP (2002) Surgery and adjunctive chemotherapy for invasive bladder cancer. Surg Oncol 11: 55-63

Rodel C, Grabenbauer GG, Kuhn R, Papadopoulos T, Dunst J, Meyer M, Schrott KM, Sauer R (2002a) Combined-modality treatment and selective organ preservation in invasive bladder cancer: long-term results. J Clin Oncol 20: $3061-3071$
Rodel C, Grabenbauer GG, Kuhn R, Zorcher T, Papadopoulos T, Dunst J, Schrott KM, Sauer R (2002b) Organ preservation in patients with invasive bladder cancer: initial results of an intensified protocol of transurethral surgery and radiation therapy plus concurrent cisplatin and 5-fluorouracil. Int J Radiat Oncol Biol Phys 52: 1303-1309

Sauer R, Birkenhake S, Kuhn R, Wittekind C, Schrott KM, Martus P (1998) Efficacy of radiochemotherapy with platin derivatives compared to radiotherapy alone in organ-sparing treatment of bladder cancer. Int $J$ Radiat Oncol Biol Phys 40: 121-127

Shipley WU, Kaufman DS, Zehr E, Heney NM, Lane SC, Thakral HK, Althausen AF, Zietman AL (2002) Selective bladder preservation by combined modality protocol treatment: long-term outcomes of 190 patients with invasive bladder cancer. Urology 60: 62 - 67 (discussion 67-8).

Shipley WU, Prout Jr GR, Kaufman SD, Perrone TL (1987) Invasive bladder carcinoma. The importance of initial transurethral surgery and other significant prognostic factors for improved survival with full-dose irradiation. Cancer 60: 514-520

Shipley WU, Winter KA, Kaufman DS, Lee WR, Heney NM, Tester WR, Donnelly BJ, Venner PM, Perez CA, Murray KJ, Doggett RS, True LD (1998) Phase III trial of neoadjuvant chemotherapy in patients with invasive bladder cancer treated with selective bladder preservation by combined radiation therapy and chemotherapy: initial results of Radiation Therapy Oncology Group 89-03. J Clin Oncol 16: 3576-3583

Stein JP, Lieskovsky G, Cote R, Groshen S, Feng AC, Boyd S, Skinner E, Bochner B, Thangathurai D, Mikhail M, Raghavan D, Skinner DG (2001) Radical cystectomy in the treatment of invasive bladder cancer: longterm results in 1,054 patients. J Clin Oncol 19: 666-675

Sternberg CN (2002) Neo-adjuvant and adjuvant chemotherapy of bladder cancer: Is there a role? Ann Oncol 13(Suppl 4): 273-279

Tester W, Caplan R, Heaney J, Venner P, Whittington R, Byhardt R, True L, Shipley W (1996) Neoadjuvant combined modality program with selective organ preservation for invasive bladder cancer: results of Radiation Therapy Oncology Group phase II trial 8802. J Clin Oncol 14: $119-126$

Tester W, Porter A, Asbell S, Coughlin C, Heaney J, Krall J, Martz K, Venner P, Hammond E (1993) Combined modality program with possible organ preservation for invasive bladder carcinoma: results of RTOG protocol 85-12. Int J Radiat Oncol Biol Phys 25: 783-790

Waehre H, Ous S, Klevmark B, Kvarstein B, Urnes T, Ogreid P, Johansen TE, Fossa SD (1993) A bladder cancer multi-institutional experience with total cystectomy for muscle-invasive bladder cancer. Cancer 72: $3044-3051$

Zietman AL, Sacco D, Skowronski U, Gomery P, Kaufman DS, Clark JA, Talcott JA, Shipley WU (2003) Organ conservation in invasive bladder cancer by transurethral resection, chemotherapy and radiation: results of a urodynamic and quality of life study on long-term survivors. J Urol 170: $1772-1776$ 